February 2006;34:93-100). (Respond: Dr Yamanouchi, Department of Pediatrics, Dokkyo University School of Medicine, 880 Kitakobayashi, Mibu, Shimotsuga, Tochigi 321-0293, Japan).

COMMENT. The differentiation of acute encephalopathy and complex febrile seizure (CFS) is often difficult, and the clinical presentation of all 9 infants with prolonged seizures and high fever highlights this controversy. Factors in favor of the diagnosis of CFS in the above series of patients include the type of infection causing the fever and seizure (influenza A, exanthem subitum, and nonspecific viral URI, all commonly associated with FS); and the elevation of interleukin- 6 cytokines, previously reported in children with FS and with influenza encephalopathy (Virta M et al. Epilepsia 2002;43:920-923; Kawada J-I et al. J Infect Dis 2003;188:690-698). The role of the cytokine response to infection in relation to the mechanism of febrile seizures is discussed in subsequent correspondence (Millichap JG, Millichap JJ. J Infect Dis 2004;189:564-565). A previous study negating an association of elevated cytokines and FS (Ichiyama T et al. Neurology 1998;50:407-411), cited by the authors of the current report, is now discounted.

The prolonged impairment of consciousness after the convulsions, and the behavioral sequelae are consistent with an encephalopathy. These cases are unique in the predilection for frontal lobe involvement, a finding that differentiates them from other acute encephalopathies of childhood, such as Reye syndrome and acute disseminated encephalomyelitis, but does not exclude a postictal pathology.

A rare case of acute necrotizing encephalopathy (ANE) associated with human herpesvirus-6 infection is reported from Sapporo, Japan (Ohsaka M et al. Pediatr Neurol Feb 2006;34:160-163). A 14-month-old infant presented with high fever and generalized tonic convulsion followed by persistent coma, rash of exanthem subitum on the $4^{\text {th }}$ day, brain swelling and herniation, the patient dying on the $12^{\text {th }}$ day of the illness. CSF protein was 73 $\mathrm{mg} / \mathrm{dL}$, cell count 2 , glucose $155 \mathrm{mg} / \mathrm{dL}$. The MRI on the $3^{\text {rd }}$ day revealed symmetric lesions in the thalamus, cerebellum, and brainstem, typical of ANE.

Cortical liquefaction is reported in a 9-month-old infant with severe HHV-6 encephalopathy (Takanashi J et al. Neurology Feb (1 of 2) 2006;66:452). MRI showing high signal intensity on T2-weighted images and low signal intensity on T1-weighted and FLAIR images in the cortex was consistent with liquefaction. Consciousness gradually improved, but the infant had severe retardation and quadriplegia.

\title{
ALEXANDER DISEASE WITHOUT WHITE MATTER LESIONS
}

Seven patients with atypical Alexander disease, showing signs of medulla and spinal cord involvement and little evidence of leukoencephalopathy, are reported from University Medical Center, Amsterdam, the Netherlands; and centers in the UK, USA, Canada, and Sweden. Patient 1 presented with progressive spinal cord dysfunction at age 9 years. Spinal MRI showed thickening of the spinal cord, and brain MRI revealed medulla and dentate nucleus changes, with a garland appearance lining the lateral ventricles. Rosenthal fibers consistent with Alexander disease were found in perivascular and subependymal regions of the ventricular wall biopsy. Diagnosis was confirmed by genetic testing and de novo mutations in the GFAP gene. All patients had juvenile onset and signs of brain stem and 
spinal cord dysfunction or atrophy; none had macrocephaly. (van der Knaap MS, Ramesh V, Schiffmann R, et al. Alexander disease. Ventricular garlands and abnormalities of the medulla and spinal cord. Neurology February (2 of 2) 2006;66:494-498). (Reprints: Dr Marje van der Knaap, Department of Child Neurology, VU University Medical Center, De Boelelaan 1117, 1081 HV Amsterdam, The Netherlands).

COMMENT. Alexander disease, a rare leukoencephalopathy caused by mutations in the GFAP gene, is characterized by white matter abnormalities, predominantly affecting the frontal lobes. The disease typically occurs in infants with macrocephaly and delayed development. Since DNA confirmation of the diagnosis, the clinical and MRI phenotype has widened to include cases with predominant brainstem and spinal cord involvement, usually of late onset. Signal abnormalities or atrophy of these locations should warrant DNA analysis for Alexander disease. An MRI appearance of ventricular garlands, a relatively new sign, is also an indication for DNA confirmation.

\section{MUSCLE DISORDERS}

\section{BRAIN MRI FINDINGS IN CONGENITAL MUSCULAR DYSTROPHY}

Brain magnetic resonance imaging (MRI) findings in 13 patients with congenital muscular dystrophy (MDCIC) and Fukutin-related protein (FKRP) gene mutations were retrospectively reviewed in a study at Hammersmith Hospital, London, UK, and European centers. MRIs were obtained between 22 months and 11 years of age. Five patients had normal MRIs and normal cognitive abilities. Three had cerebellar cysts and cognitive impairment (IQ 50, 56, and 70); 1 was microcephalic. Five had cerebellar cysts associated with other structural abnormalities, including nodular heterotopia, frontal pachygyria, pontine hypoplasia, microcephaly, lissencephaly, Dandy-Walkerlike malformation, and absence of cerebellar vermis. One patient had Walker-Warburg syndrome (WWS) with involvement of muscle, eye and brain. The severity of CNS involvement reflected the severity of disruption of a-dystroglycan glycosylation (DGG). DGG was almost absent in the patient with WWS and less severely reduced in patients with MDCIC with or without cerebellar cysts. (Mercuri E, Topaloglu H, Brockington M, et al. Spectrum of brain changes in patients with congenital muscilar dystrophy and FKRP gene mutations. Arch Neurol Feb 2006;63:251-257). (Respond: Francesco Muntoni MD, Dubowitz Neuromuscular Centre, Department of Paediatrics, Imperial College, Hammersmith Hospital Campus, Du Cane Road, London WI2 OHN, UK).

COMMENT. Only 5 of 13 patients with congenital muscular dystrophy and FKRP gene mutations had normal brain MRIs and normal IQs. Eight patients had structural brain anomalies consisting of cerebellar cysts with or without other structural changes and cognitive deficits of various degrees of severity. Cerebellar cysts, either alone or associated with vermis hypoplasia and white matter abnormalities, were the most common MRI findings with FKRP mutations and MDCIC. The degree of abnormal glycosylation of adystroglycan correlates with disease severity, MDCIC having a more severe depletion compared with later onset limb girdle MD. Congenital MDs are called dystroglycanopathies and include Fukuyama CMD, muscle-eye-brain disease, and Walker-Warburg syndrome. 